\title{
Effects of reward magnitude on running speed following a deprivation upshift
}

\author{
T. L. DAVIDSON, ELIZABETH D. CAPALDI, and DAVID E. MYERS \\ Purdue University, West Lafayette, Indiana 47907
}

The running speed of rats trained in a straight alley under low deprivation $199 \%-101 \%$ ad-lib body weight) and then shifted to high deprivation (79\%-81\% ad-lib body weight) was compared with that of unshifted high-deprivation controls. Running speeds of shifted rats increased more rapidly, relative to unshifted controls, when reward magnitude was large (22 $.045-\mathrm{g}$ pellets) as compared with small (2 .045-g pellets). Thus the effect of a deprivation shift on instrumental performance was found to vary as a function of reward magnitude. Deprivation downshifts also produce more rapid and larger performance changes when large reward is used than when small reward is used. It seems that responsivity to changes in level of deprivation is an increasing function of reward magnitude.

Is the effect of a shift in deprivation level dependent upon reward magnitude? With respect to downshifts in deprivation, this question has been answered affirmatively. Capaldi (1973) found that, following extended runway training under high deprivation, rats shifted to low deprivation showed a larger, more rapid decrement in running speed, relative to unshifted low-deprivation controls, when large rather than small reward was employed. The present experiment is the deprivationupshift analogue of the Capaldi (1973) study. The question of experimental interest is whether or not the effects of a shift from low to high deprivation are also dependent upon reward magnitude.

This question is an important one. Capaldi (1973) suggested two plausible general approaches to how instrumental performance following a deprivation shift is influenced by reward magnitude. One way in which these views can be distinguished is on the basis of the differential predictions they make concerning the effects of reward magnitude on instrumental behavior following an upshift in deprivation level.

One account suggests that differences between the internal stimulus concomitants of high and low deprivation are accentuated as an increasing function of reward magnitude. Hence, the decremental effects of the change in stimulation produced by upshifts or downshifts in deprivation level would be greater with large as compared with small reward. Therefore, following an upshift in deprivation, rats trained with large reward would not be expected to increase in running speed as rapidly as rats trained with small reward.

Alternatively, Capaldi (1973) also considered the possibility that responsivity to any change in deprivation level is greater when training is with large rather than

This research was supported in part by a Purdue Research Foundation David Ross Grant. Reprint requests should be sent to T. L. Davidson, Department of Psychological Sciences, Purdue University, West Lafayette, Indiana 47907. small reward. This notion suggests that the extent to which instrumental performance is decremented by decreasing deprivation, or enhanced by increasing deprivation, is a positive function of reward magnitude. Viewed in this way, it would be expected that rats trained with large reward would increase in running speed more rapidly than rats trained with small reward, following an upshift in level of deprivation.

Clearly, these accounts are in direct opposition, with respect to their expectations regarding the effects of reward magnitude on instrumental performance following an upshift in level of deprivation. The present experiment tested these competing views.

\section{METHOD}

\section{Subjects}

The subjects were 44 naive male albino rats, about 90 days old upon arrival from the Holtzman Company, Madison, Wisconsin.

\section{Apparatus}

The apparatus was a runway painted a flat gray throughout, $190.5 \times 10.2 \mathrm{~cm}$ with $22.9-\mathrm{cm}$ high sides covered with $1.3-\mathrm{cm}$ hardware cloth. A $20.3-\mathrm{cm}$ tong floor treadle suspended over a microswitch constituted the initial portion of the alley. When the treadle was depressed by the weight of the rat, a $.01 \mathrm{sec}$ clock was started. This clock was stopped (start time) and another started when the rat interrupted a photobeam $13.0 \mathrm{~cm}$ beyond the treadle. The latter clock was stopped (run time) and a third one started when the rat broke the second photobeam, located $106.7 \mathrm{~cm}$ from the first. Interruption of the third photobeam stopped the final clock (goal time). The three times were summed to obtain a total time. The third photobeam was $38.7 \mathrm{~cm}$ beyond the second photobeam and $7.5 \mathrm{~cm}$ in front of a gray block of wood with a hole $3.17 \mathrm{~cm}$ in diameter and $1.9 \mathrm{~cm}$ deep, which served as a food cup. A manually lowered guillotine door $36.8 \mathrm{~cm}$ from the rear of the goal compartment served to confine the rat to the goal area.

\section{Procedure}

The rats were fed ad lib for 11 days following arrival at the laboratory. Four groups were formed matched on original ad lib 
weight (mean weight for each rat over the last 3 of these days). The groups were designated by the reward magnitude they received throughout training ( 2 or $22.045-\mathrm{g}$ Noyes pellets) and their Phase 1/Phase 2 deprivation levels. Groups $2 \mathrm{~L}-\mathrm{H}$ and $22 \mathrm{~L}-\mathrm{H}$ were trained under low deprivation (99\%-101\% of original ad-lib body weight); Groups $2 \mathrm{H}-\mathrm{H}$ and $22 \mathrm{H}-\mathrm{H}$ were trained under high deprivation $(79 \%-81 \%$ of original ad lib body weight) in both Phases 1 and 2 . The amount of food given each rat was rationed to achieve the percentage body weight appropriate to its designated deprivation level. These weight ranges were reached in 9 days. On Days 10 and 11 , each rat was handled for $90 \mathrm{sec}$. Fifteen minutes after handling, the rats in Groups $22 \mathrm{H}-\mathrm{H}$ and $22 \mathrm{~L}-\mathrm{H}$ were fed $22.045 \mathrm{~g}$ pellets, and rats in Groups $2 \mathrm{H}-\mathrm{H}$ and $2 \mathrm{~L}-\mathrm{H}$ were fed two $.045 \mathrm{~g}$ pellets in their home cages. Phase 1 began on Day 12. On Day 1 of Phase 1 each rat received one trial, on Day 2 each rat received two trials, and on Days 3-26, each rat received three trials, for a total of 75 Phase 1 trials. At the end of Phase 1 , the rats under low deprivation (99\%-101\% original ad-lib body weight) were shifted to high deprivation ( $79 \%-81 \%$ original ad lib body weight). This shift required 12 days to complete. Phase 2 began on the following day, with each rat receiving 3 trials/day for 10 days with the same reward magnitude as given in Phase 1 .

Throughout the study rats were run in squads of four, composed of one subject from each group, yielding an intertrial interval of 4-7 min. On each trial a rat was allowed $60 \mathrm{sec}$ to traverse each alley section before being gently guided to the next section, with $60 \mathrm{sec}$ recorded for each untraversed segment. Each rat was removed from the goalbox immediately after the entire reward was consumed. Each squad was fed its daily ration $15 \mathrm{~min}$ after completing its trials. Prior to the conclusion of the experiment, two rats, one each from Groups $2 \mathrm{~L}-\mathrm{H}$ and $22 \mathrm{~L}-\mathrm{H}$ became ill, and their data were discarded.

\section{RESULTS}

All running times were converted to speeds $(1 / \mathrm{sec})$. Speeds in Phases 1 and 2 were analyzed in a 2 by 2 between-within analysis of variance for unequal ns, with reward magnitude and Phase 1 deprivation level as factors. Although differences were largest in the start, the general pattern of results for the shifted groups was essentially the same in each alley section; thus, only total speeds are reported.

Figure 1 shows mean total speeds for each group on

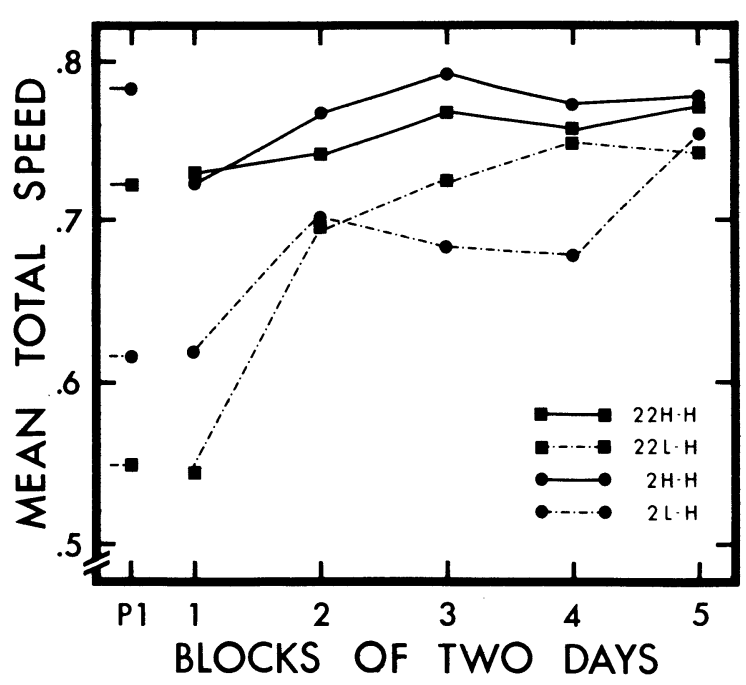

Figure 1. Mean total speed for each group on the last 2-day block of Phase 1 and each 2-day block of Phase 2. the last 2-day block of Phase 1 and on each 2-day block of Phase 2. Over all of Phase 1 (excluding Day 1), rats under high deprivation ran faster than those under low deprivation $[\mathrm{F}(1,38)=34.42, \mathrm{p}<.01]$. This difference grew smaller over blocks [Phase 1 Deprivation by Blocks, $F(11,418)=4.45, p<.01]$. Reward magnitude produced no significant main effects or interactions with blocks or Phase 1 deprivation level over all of Phase 1 . The finding of no acquisition differences as a function of reward magnitude is not unusual (e.g., Gonzalez, Gleitman, \& Bitterman, 1962; Mellgren, 1972).

In Phase 2, Figure 1 shows that the running speeds of groups shifted from low to high deprivation gradually increased to the level of the unshifted controls. As also can be seen in Figure 1, the shifted group given large reward (Group 22L-H) reached the level of its control (Group 22H-H) more quickly than did the shifted group given small reward (Group 2L-H vs. Group 2H-H). These differences were statistically reliable. In Phase 2, there was a significant effect of Phase 1 deprivation level $[F(1,38)=4.20, p<.05]$, with this difference decreasing over blocks [Phase 1 Deprivation Level by Blocks, $F(4,152)=4.95, p<.01]$, differentially as a function of reward magnitude [Phase 1 Deprivation Level by Reward Magnitude by Blocks, $F(4,152)=2.80, p<.05]$. Newman-Keuls tests showed that Group 2L-H ran significantly slower than Group 2H-H until Block 5 (ps <.05), while Group $22 \mathrm{~L}-\mathrm{H}$ ran significantly slower than Group $22 \mathrm{H}-\mathrm{H}$ only on Block $1(\mathrm{p}<.01)$.

\section{DISCUSSION}

The present study showed that the effects of a shift to high deprivation, following extended training under low deprivation, varied as a function of reward magnitude. Running speeds of rats shifted from low to high deprivation increased more rapidly, relative to unshifted high-deprivation controls, when reward magnitude was large as compared with small. These findings complement those of Capaldi (1973), which showed that the decremental effects of a downshift in deprivation level were also greater with large than with small reward. As noted earlier, two plausible, but competing accounts of how instrumental performance following deprivation shifts is influenced by reward magnitude were suggested by Capaldi's (1973) findings. The results of the present experiment provide a basis for evaluating these interpretations.

One possibility was that with large reward the change in stimulation produced by a deprivation shift is greater than it is with small reward. This notion can be rejected on the basis of the present findings. According to this view, differences between the internal stimulus concomitants of high and low deprivation are accentuated as an increasing function of reward magnitude. Hence, due to differential stimulus generalization decrement, the upshift performance of rats trained with large reward would not improve as rapidly as that for rats trained with small reward. Contrary to this expectation, the present experiment showed that instrumental responding following an upshift in deprivation improved more rapidly when large as compared with small reward was employed.

Instead, the results of the present study and those of Capaldi (1973) suggest that general responsivity to any change in deprivation level (i.e., increases or decreases) is greater when training occurs with large rather than small reward. Considered together, the findings of Capaldi (1973) and those of the present experi- 
ment show that the degree to which instrumental responding is decremented when deprivation is decreased or enhanced when deprivation is increased is a direct function of reward size. In general, it appears that the larger the reward magnitude, the greater are the behavioral effects of changes in deprivation level.

A number of mechanisms can potentially account for these effects. For example, if it is assumed that both reward magnitude and deprivation level contribute to incentive motivation (e.g., Bolles, 1975; Mollenauer, 1971), it is possible that the effects of reward magnitude on instrumental performance following deprivation shifts are attributable to incentive differences. Specifically, the results of the present study and those of Capaldi (1973) are consistent with the notion that increases in incentive produced by deprivation upshifts and decreases in incentive resulting from downshifts in deprivation are both greater with large than with small reward.

Alternatively, reward magnitude may affect responsivity to deprivation changes through the operation of associative mechanisms. There is evidence that the stimulus consequences of deprivation elicit internal representations (i.e., memories, expectancies, or anticipations) of reward, which in turn elicit instrumental responses (e.g., Capaldi, Smith, \& White, 1977; Peck \& Ader, 1974). It is possible that such internal reward representations are strengthened as a result of deprivation upshifts and weakened as a result of downshifts in deprivation level, with both effects more pronounced when large reward is employed.

A final point of interest concerns the similarity between the effects of reward magnitude on shifts in deprivation level and the effects of deprivation level on shifts in reward magnitude. Ehrenfreund (1971) found that instrumental responding was more greatly incremented by increases in reward size and more greatly decremented by decreases in reward magnitude under high deprivation than under low deprivation. Hence, it seems that not only is responsivity to changes in deprivation level dependent upon reward magnitude, but responsivity to changes in reward magnitude is dependent upon deprivation level. Whether or not these parallel findings are best accounted for by common underlying associative or incentive mechanisms such as those described above, or by a different common mechanism, or by completely independent mechanisms is a question requiring further investigation.

\section{REFERENCES}

Bolles, R. C. Theory of motivation. New York: Harper \& Row, 1975.

Capaldi, E. D. Effects of shifts in body weight on rats' straight alley performance as a function of reward magnitude. Learning and Motivation, 1973, 4, 229-235.

Capaldi, E. D., Smith, N. S., \& White, L. A. Control of reward expectancies by drive stimuli. Journal of Experimental Psychology: Animal Behavior Processes, 1977, 3, 229-235.

Ehrenfreund, B. Effect of drive on successive magnitude shift in rats. Journal of Comparative and Physiological Psychology, 1971, 76, 418-423.

Gonzalez, R. C., Gleitman, H., \& Bitterman, M. E. Some observations on the depression effect. Journal of Comparative and Physiological Psychology, 1962, 55, 578-581.

Mellgren, R. L. Positive and negative contrast effects using delayed reinforcement. Learning and Motivation, 1972, 3, 185-193.

Mollenauer, S. O. Shifts in deprivation level: Different effects depending on amount of preshift training. Learning and Motivation, 1971, 2, 58-60.

PECK, J. H., \& Ader, R. Illness-induced taste aversion under states of deprivation and satiation. Animal Learning \& Behavior, 1974, 2, 6-8.

(Received for publication December 11, 1979.) 